\title{
RESEARCH
}

\section{Explaining Pharmacy Students' Dispensing Intentions in Substance Abuse- Related Gray Areas Using the Theory of Planned Behavior}

\author{
KariLynn Dowling-McClay, PharmD, MPH, ${ }^{\mathrm{a}}$ Cortney M. Mospan, PharmD, ${ }^{\mathrm{b}}$ Pooja Subedi, MPH, \\ Nicholas E. Hagemeier, PharmD, $\mathrm{PhD}^{\mathrm{a}}$ \\ ${ }^{a}$ East Tennessee State University Bill Gatton College of Pharmacy, Johnson City, Tennessee \\ ${ }^{\mathrm{b}}$ Wingate University School of Pharmacy, Wingate, North Carolina \\ ${ }^{c}$ East Tennessee State University College of Public Health, Johnson City, Tennessee
}

Submitted August 30, 2017; accepted April 9, 2018; published June 2019.

Objective. To examine the extent to which theory of planned behavior (TPB) constructs and demographic characteristics explain pharmacy students' dispensing intentions in ethically or legally gray areas involving potential substance misuse or abuse.

Methods. Two cohorts of third-year Doctor of Pharmacy (PharmD) students $(n=159)$ were provided with five written cases describing common "gray area" dispensing scenarios in community practice involving medications and devices with potential for misuse or abuse (eg, long-term buprenorphine maintenance prescription without evidence of tapering, early refill of a narcotic for an out-of-town patient, non-prescription sale of pseudoephedrine). Students completed a 12-item survey instrument for each case. Items assessed whether the student would dispense the medication or device in the given scenario, how many times in 10 similar scenarios the student would dispense the medication or device, attitudes regarding dispensing, and subjective norm and perceived behavioral control beliefs.

Results. Wide variation in the percentages of students who would dispense the medications or devices was noted across the five scenarios (14\% in the buprenorphine scenario to $61 \%$ in the pseudoephedrine scenario). Attitude scores significantly predicted dispensing decisions in all scenarios $(p<.001)$, whereas subjective norm and perceived behavioral control beliefs were significant predictors of dispensing only in select case scenarios. Gender and community pharmacy work experience did not consistently predict dispensing intentions.

Conclusion. Student attitudes consistently predicted intent to dispense across five gray practice scenarios. These findings can be used to inform development of educational interventions that influence students' attitudes and self-awareness in community practice decision-making scenarios involving potential substance misuse or abuse.

Keywords: pharmacy student, theory of planned behavior, dispensing, substance abuse

\section{INTRODUCTION}

In 2017, 18.1 million Americans 12 years of age or older reported past-year misuse of prescription drugs. ${ }^{1}$ As nationwide substance misuse (any use outside of a prescriber's direction) and abuse (a symptom of substance use disorder) escalates, so do associated outcomes of infectious disease transmission, overdose, and death. ${ }^{1-3}$ In response to the public health crisis, an "all hands on deck" approach requires pharmacists to work alongside other health professionals in attempts to reverse the trend. ${ }^{4}$ In the Surgeon General's Report on Alcohol, Drugs, and Health, pharmacists are called to engage more in sub-

Corresponding Author: KariLynn Dowling-McClay, East Tennessee State University Bill Gatton College of Pharmacy, Box 70657, Johnson City, TN 37614. Tel: 423-439-6752. Fax: 423-439-6784. E-mail: dowlingk1@etsu.edu. stance misuse and abuse prevention and treatment. ${ }^{3}$ The American Society of Health-System Pharmacists has issued multiple statements regarding the pharmacist's role in substance abuse prevention, education, and treatment. ${ }^{5}$ Likewise, the American Association of Colleges of Pharmacy (AACP) has published curricular guidelines specific to the disease of addiction and encouraged schools and colleges to publicly commit to educating pharmacy students regarding the prevention and treatment of substance abuse. .,7 $^{6}$

Ethically and legally gray areas are common in community pharmacy settings, and perhaps more so in scenarios that involve medications with potential for misuse or abuse. A "gray area," as defined by the authors, is a practice scenario in which there is no single, correct clinical decision and no legally mandated behavior under federal 


\section{American Journal of Pharmaceutical Education 2019; 83 (5) Article 6767.}

and state regulations. Despite a growing body of research examining pharmacists' attitudes and patient care behaviors regarding substance abuse, research specific to student pharmacists' substance abuse perceptions and behaviors is limited. ${ }^{8-11}$ When examining pharmacy students' pre-intervention perceptions about alcoholism, Neville found that students tended to report neutral attitudes toward role adequacy, self-efficacy, motivation, role legitimacy, and work satisfaction specific to alcohol use disorders. ${ }^{12}$ Richard and colleagues also noted neutral student attitudes toward addiction and limited student knowledge about addiction. ${ }^{13}$ Additional research among community pharmacists and primary care prescribers has noted significant variation in attitudes and patient care behaviors specific to prescription drug abuse. ${ }^{14-17}$ There is value in assessing student pharmacists' perceptions and intentions to dispense medications in gray areas of practice because opportunities exist to intervene via didactic and experiential education to influence students' decisionmaking processes before they are solidified in practice. To the authors' knowledge, this is the first theory-based study to examine pharmacy students' anticipated patient care behaviors and decision-making specific to substance abuse-related scenarios.

Behavioral theories are instrumental in understanding human actions, including dispensing of prescriptions to patients by pharmacists. One well-established theory, the Theory of Planned Behavior (TPB), postulates that planning to perform a behavior (behavioral intention) and actual engagement in a behavior are predicted by three constructs: an individual's attitude toward the behavior, perceived social pressures from peers' expectations and behaviors (subjective norms), and perceived control over one's own ability to engage in the behavior (Figure 1). ${ }^{18}$ Generally, the more favorable attitudes and subjective norm beliefs are toward a behavior, and the greater perceived control there is over the behavior, the more likely an individual is to engage in a specific behavior when an opportunity arises. In ethically and legally gray areas, use of the TPB is useful in determining the primary drivers of behavior. The TPB has been used to explain many health care provider behaviors, including communication about misuse and abuse of opioid pain relievers, intention to educate patients regarding medication disposal, and intention to use a prescription drug monitoring program. ${ }^{8-9,15,19}$ Importantly, use of a theoretical framework such as the TPB informs behavior-specific intervention development. The objective of this study was to use the TPB to examine the extent to which pharmacy students' attitudes, subjective norm beliefs, perceived behavioral control beliefs, gender, and community pharmacy work experience predict substance abuse-related dispensing behaviors.

\section{METHODS}

This study protocol was determined to be exempt from human research status by the East Tennessee State University Institutional Review Board. In April 2016 and January 2017, third-year (P3) Doctor of Pharmacy (PharmD) students ( $n=83$ and $n=76$, respectively) were provided five brief, written community pharmacy case scenarios at the beginning of a four-hour integrated skills laboratory. The cases described scenarios in which patients requested medications or medical devices with potential for misuse or abuse, and were written from the second-person point of view, placing the reader as the pharmacist. Cases included: a long-term buprenorphine maintenance prescription without evidence of tapering or counseling, early refill of a narcotic for an out-of-town patient, new prescriptions from a pain management clinic

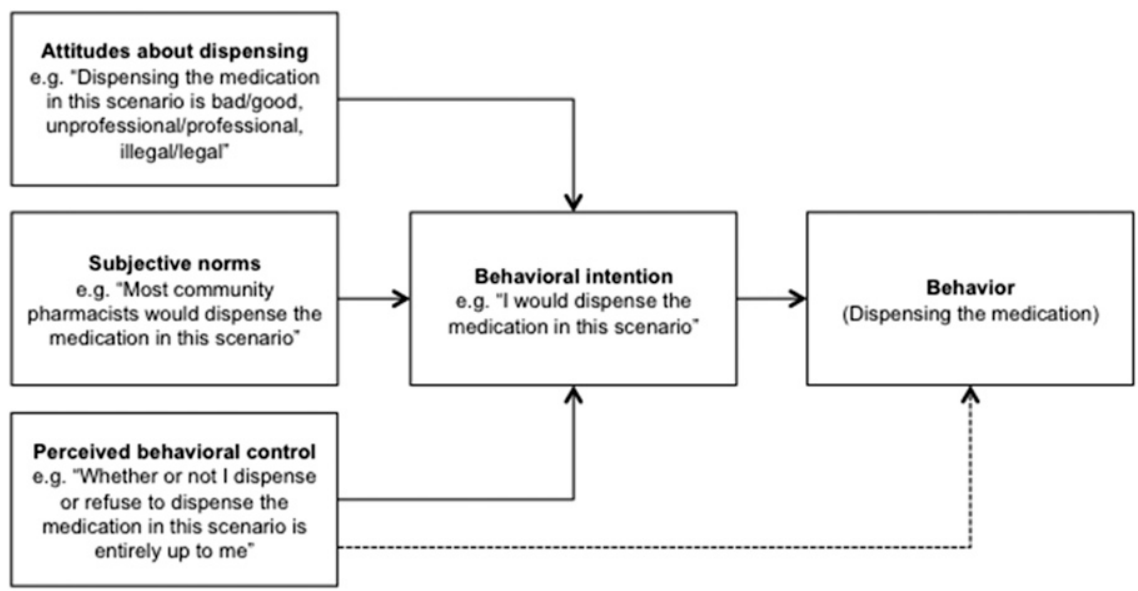

Adapted from Ajzen I. The Theory of Planned Behavior. Organizational Behavior and Human Decision Processes. 1991;50:179-211.

Figure 1. The Theory of Planned Behavior Applied to Pharmacy Students’ Dispensing Decisions 


\section{American Journal of Pharmaceutical Education 2019; 83 (5) Article 6767.}

for a patient known to the pharmacy, non-prescription sale of syringes, and non-prescription sale of pseudoephedrine. The cases were developed based on community practice experiences of the authors and data gathered through a previously conducted qualitative study involving pharmacists. ${ }^{17}$

At the beginning of the laboratory period, students were instructed to independently read the cases and complete anonymous, 12-item, TPB-based paper survey instruments for each scenario. ${ }^{18,20}$ To evaluate behavioral intention, students were asked to indicate, as the pharmacist in the scenario, if they would dispense or not dispense the medication/device, and how many times out of 10 similar scenarios they would dispense the medication/device. Standard TPB seven-point response scales were used to evaluate attitudes regarding dispensing (eg, bad-good, unprofessional-professional, illegal-legal) and level of agreement (strongly disagree-strongly agree) with subjective norm and perceived behavioral control statements. Student gender and community pharmacy work experience outside of required curricular experiences were also collected.

After all students read each case and completed the case-specific surveys, the remainder of the laboratory period was devoted to discussing each case among peers under the guidance of two faculty facilitators. The objective of the laboratory activity and resulting discussion was for students to reflect on their initial "gut" reactions and gain critical-thinking experience and self-awareness in applying decision-making to common practice scenarios where the appropriate action to take was in a gray area in terms of established ethico-legal principles and considerations for the patient's best interest.

Descriptive statistics were calculated for all survey items. Composite scores were calculated for the attitude, subjective norm, and perceived behavioral control constructs by taking the average of the responses for relevant items. One item developed to evaluate the extent to which engaging in the behavior is easy or hard was omitted from the perceived behavioral control construct to maximize internal consistency reliability. Cronbach's alpha coefficients for the final constructs applied to each scenario ranged from 0.77 to 0.89 for attitude, 0.66 to 0.81 for subjective norm beliefs, and 0.26 to 0.63 for perceived behavioral control beliefs.

A logistic regression model was used to examine the extent to which student gender, work experience, cohort, attitude, subjective norm beliefs, and perceived behavioral control beliefs predicted the dispensing decision in each scenario. An alpha level of $p=.05$ was set a priori. Likewise, a negative binomial regression model was used to examine the extent to which the same independent variables explained mean intent to dispense in 10 similar sce- narios. This regression model was used instead of Poisson regression due to over dispersal of the outcome variable for some of the scenarios. All analyses were completed using SAS, Version 9.4 (SAS Institute, Inc, Cary, NC).

\section{RESULTS}

A $100 \%$ response rate was obtained. Approximately half of the study population (49\%) were female students and a majority (78\%) had co-curricular community pharmacy work experience. Students' reported dispensing decisions, mean intent to dispense in similar scenarios, and mean TPB construct scores are reported in Table 1. The extent to which students indicated they would dispense the medication or device ranged from $14 \%$ in the buprenorphine scenario to $61 \%$ in the pseudoephedrine scenario. Mean intent to dispense in 10 similar scenarios ranged from 2.3 (early narcotic refill) to 5.1 (pseudoephedrine). The TPB construct scores for the scenarios ranged from 2.9 to 4.4 for attitude, 3.1 to 4.5 for subjective norm, and 5.5 to 6.0 for perceived behavioral control.

Results of the logistic regression analyses are reported in Table 2. In all scenarios, more positive attitudes toward the behavior were significantly associated with the decision to dispense the medication/device $(p<.001)$, with adjusted odds ratios (aOR) ranging from 3.26 (95\% CI $1.91-5.59)$ in the buprenorphine scenario to 8.42 (95\% CI 3.80 -18.64) in the pain clinic scenario. Higher subjective norm scores were associated with the decision to dispense in the early refill scenario $(\mathrm{aOR}=2.08 ; 95 \%$ CI $1.12-3.86 ; p=.02)$ and the pseudoephedrine scenario $(\mathrm{aOR}=1.14 ; 95 \%$ CI 1.13 - 4.06; $p=.008$ ). Greater perceived behavioral control was negatively associated with dispensing decision in the pseudoephedrine scenario $(\mathrm{aOR}=0.46 ; 95 \%$ CI $0.27-0.80$; $p=.005$ ). Student gender, prior work experience, and cohort membership did not significantly predict the decision to dispense in any scenario.

The mean intent to dispense output resulting from negative binomial regression analyses are presented in Table 3. In all scenarios, increases in attitude scores were significantly associated with increased mean intent to dispense the medication/device in similar scenarios $(p<.001)$. For the pseudoephedrine scenario, prior work experience $(p=.003)$ and greater subjective norm scores $(p=.002)$ were also significantly associated with increased dispensing intention. Higher perceived behavioral control scores were associated with lower dispensing intention in the pseudoephedrine $(p=.04)$ and pain management clinic $(p=.02)$ scenarios. Unlike all other scenarios, male students reported significantly higher mean intent to dispense in the early narcotic refill scenario compared to female students $(p=.03)$. 


\section{American Journal of Pharmaceutical Education 2019; 83 (5) Article 6767.}

Table 1. Pharmacy Students' Self-Reported Dispensing Decisions in Substance Abuse-Related Gray Areas of Pharmacy Practice and Descriptive Statistics for Theory of Planned Behavior Constructs $(\mathrm{N}=159)^{\mathrm{a}}$

\begin{tabular}{|c|c|c|c|c|c|c|}
\hline \multirow[b]{2}{*}{ Scenario } & \multicolumn{2}{|c|}{$\begin{array}{l}\text { Dispensing } \\
\text { Decision }\end{array}$} & \multirow{2}{*}{$\begin{array}{l}\text { Intent to Dispense in } 10 \\
\text { Similar Scenarios, } \\
M(S D)\end{array}$} & \multirow{2}{*}{$\frac{\text { Attitude, }}{\text { M (SD) }}$} & \multirow{2}{*}{$\begin{array}{c}\begin{array}{c}\text { Subjective } \\
\text { Norm, }\end{array} \\
\text { M (SD) }\end{array}$} & \multirow[b]{2}{*}{$\frac{\text { Perceived Behavioral Control, }}{\text { M (SD) }}$} \\
\hline & $\begin{array}{c}\text { Yes, } \\
\text { No. }(\%)\end{array}$ & $\begin{array}{c}\text { No, } \\
\text { No. }(\%)\end{array}$ & & & & \\
\hline Buprenorphine & $22(14)$ & $137(86)$ & $2.6(3.2)$ & $3.0(1.3)$ & $3.6(1.6)$ & $6.0(1.2)$ \\
\hline Early Refill & $28(18)$ & $129(82)$ & $2.3(3.3)$ & 2.9 (1.6) & $3.1(1.5)$ & $5.7(1.2)$ \\
\hline Pain Clinic & $49(31)$ & $110(69)$ & $3.8(3.5)$ & $3.7(1.5)$ & $4.4(1.5)$ & $5.5(1.4)$ \\
\hline Syringe & $75(48)$ & $82(52)$ & $4.9(3.8)$ & $4.0(1.5)$ & $3.2(1.4)$ & $5.6(1.3)$ \\
\hline Pseudoephedrine & $97(61)$ & $62(39)$ & $5.1(3.3)$ & $4.4(1.5)$ & $4.5(1.5)$ & $5.6(1.2)$ \\
\hline
\end{tabular}

${ }^{\mathrm{a}}$ Because of case scenario-specific incomplete response rates, dispensing decisions do not add up to 159 in all case scenarios

\section{DISCUSSION}

Given the extent to which ethically and legally gray decision-making occurs in community pharmacy practice, the large number of pharmacy graduates who enter these positions, and the prevalence of substance misuse and abuse, a case-based laboratory was developed to expose students to hypothetical situations involving potential substance misuse or abuse that are likely to be encountered in practice. After completing the TPB survey instruments during the laboratory session, students routinely shared beliefs and judgments that constructed their attitudes but made few comments about their perceptions of other pharmacists or the extent to which dispensing is under their control. Therefore, it was not surprising that students' attitudes were the only TPB construct that strongly and consistently predicted dispensing decisions across all scenarios. This finding is important to consider when providing learners with opportunities to self-reflect on their behaviors and practice intentions. Attitudes and the perceptions that form them are modifiable. Interestingly, mean attitude scores were negative or neutral at best in all scenarios.

Students were least willing to dispense in the buprenorphine case and most willing to dispense in the two scenarios involving non-prescription product sales (pseudoephedrine and syringes). When discussing the buprenorphine case, students indicated they were not comfortable with certain aspects of the case, including the type of product prescribed, the patient's responses to the pharmacist's questions, and the appropriateness of medication-assisted treatment (MAT) in the management of substance use disorders. All students had completed the Neurologic and Psychiatric Pharmacy and Pharmacy Law and Ethics components of the curriculum prior to participating in this study. Overall, students' perceptions about and discomfort with buprenorphine mirrored anecdotal evidence from the authors' ongoing work with community pharmacists. Stated succinctly, students' mistrust of the prescriber and/or MAT informed their attitudes regarding buprenorphine dispensing.

Regarding OTC products, perhaps students could relate more to an activity in which they may have participated (eg, purchasing pseudoephedrine-containing products) or felt that the purchase of pseudoephedrine, if inappropriate, would be detected when submitted electronically to the National Precursor Log Exchange (NPLEx), the real-time methamphetamine precursor monitoring system used in Tennessee. Decision-making may have been influenced by individual students' interpretations of vague laws, such as the Tennessee syringe regulations requiring "proof of medical need" for nonprescription sales. ${ }^{21}$ From a population health standpoint, some students mentioned that dispensing syringes (ie, potentially providing clean needles to individuals with substance use disorders) was a harm reduction approach that factored in to their dispensing decision.

Overall, the complexity inherent in the cases and the manner in which they were presented encouraged students to "go with your gut" when deciding whether or not to dispense. The AACP's report, titled Substance Abuse: The Pharmacy Educator's Role in Prevention and Recovery, states, "Students should be exposed to the ethical and moral issues involved in the area of addiction and related disorders. They should be given the opportunity to reflect on their perceptions of the addict, and the addict's impact on society as a whole, and on other individuals specifically."6 One of the goals when developing cases for use in the laboratory was to expose students to authentic scenarios in which their default reactions and perceptions may not have been in the best interest of the patient. Ajzen posits that attitudes consist of two components: beliefs about the consequences of a behavior and positive or negative judgments regarding the consequences. ${ }^{18}$ A responsibility of the pharmacy educator is to teach students how to approach gray areas of practice, to the extent possible, in an evidence-based 


\section{American Journal of Pharmaceutical Education 2019; 83 (5) Article 6767.}

Table 2. Output From Logistic Regression Showing Factors That Influence Pharmacy Students' Self-Reported Dispensing Decisions in Substance Abuse-Related Gray Areas of Pharmacy Practice $(\mathrm{N}=159)$

\begin{tabular}{|c|c|c|c|c|c|}
\hline Variables & 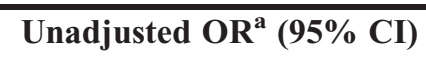 & $p$ Value & Adjusted OR ${ }^{\mathrm{b}}(95 \%$ CI) & $p$ Value & R-square \\
\hline \multicolumn{6}{|l|}{ Early Refill Scenario } \\
\hline Gender (male vs female) & $2.28(0.96-5.43)$ & .06 & $1.69(0.41-6.87)$ & .47 & 0.44 \\
\hline Prior work experience (yes vs no) & $1.94(0.62-6.02)$ & .25 & $3.27(0.47-22.52)$ & .23 & \\
\hline Cohort (2016 vs 2017) & $2.88(1.19-6.99)$ & .02 & $2.09(0.49-8.92)$ & .32 & \\
\hline Attitude & $5.15(2.93-9.05)$ & $<.001$ & $3.60(1.99-6.54)$ & $<.001$ & \\
\hline Subjective norm & $3.37(2.17-5.22)$ & $<.001$ & $2.08(1.12-3.86)$ & .02 & \\
\hline Perceived behavioral control & $0.57(0.41-0.81)$ & .002 & $0.57(0.30-1.08)$ & .08 & \\
\hline \multicolumn{6}{|l|}{ Buprenorphine Scenario } \\
\hline Gender (male vs female) & $1.53(0.61-3.83)$ & .36 & $2.26(0.71-7.12)$ & .16 & 0.24 \\
\hline Prior work experience (yes vs no) & $2.07(0.58-7.44)$ & .27 & $0.75(0.15-3.74)$ & .73 & \\
\hline Cohort (2016 vs 2017) & $0.73(0.30-1.8)$ & .50 & $0.77(0.25-2.40)$ & .65 & \\
\hline Attitude & $3.59(2.16-5.96)$ & $<.001$ & $3.26(1.91-5.59)$ & $<.001$ & \\
\hline Subjective norm & $1.65(1.19-2.29)$ & .002 & $1.33(0.85-2.08)$ & .21 & \\
\hline Perceived behavioral control & $1.09(0.72-1.63)$ & .70 & $1.16(0.64-2.09)$ & .62 & \\
\hline \multicolumn{6}{|l|}{ Pain Clinic Scenario } \\
\hline Gender (male vs female) & $1.25(0.63-2.47)$ & .52 & $1.41(0.50-3.94)$ & .52 & 0.46 \\
\hline Prior work experience (yes vs no) & $0.94(0.43-2.07)$ & .89 & $0.64(0.15-2.62)$ & .53 & \\
\hline Cohort (2016 vs 2017) & $1.50(0.76-2.97)$ & .24 & $0.50(0.17-1.54)$ & .23 & \\
\hline Attitude & $7.44(3.84-14.41)$ & $<.001$ & $8.42(3.80-18.64)$ & $<.001$ & \\
\hline Subjective norm & $2.17(1.6-2.95)$ & $<.001$ & $1.29(0.80-2.07)$ & .29 & \\
\hline Perceived behavioral control & $0.774(0.58-9.95)$ & .02 & $0.66(0.44-0.99)$ & .05 & \\
\hline \multicolumn{6}{|l|}{ Syringe Scenario } \\
\hline Gender (male vs female) & $1.44(0.77-2.71)$ & .26 & $0.90(0.33-2.43)$ & .83 & 0.49 \\
\hline Prior work experience (yes vs no) & $0.95(0.44-2.05)$ & .89 & $0.73(0.20-2.63)$ & .63 & \\
\hline Cohort (2016 vs 2017) & $2.13(1.13-4.03)$ & .02 & $0.98(0.34-2.84)$ & .97 & \\
\hline Attitude & $7.14(3.86-13.19)$ & $<.001$ & $7.34(3.85-14.02)$ & $<.001$ & \\
\hline Subjective norm & $1.25(0.99-1.57)$ & .06 & $1.09(0.75-1.57)$ & .65 & \\
\hline Perceived behavioral control & $0.86(0.68-1.10)$ & .22 & $1.10(0.73-1.66)$ & .64 & \\
\hline \multicolumn{6}{|l|}{ Pseudoephedrine Scenario } \\
\hline Gender (male vs female) & $1.31(0.69-2.50)$ & .41 & $2.84(0.83-9.67)$ & .95 & 0.56 \\
\hline Prior work experience (yes vs no) & $1.02(0.47-2.21)$ & .96 & $0.99(0.23-4.39)$ & $>.99$ & \\
\hline Cohort (2016 vs 2017) & $0.35(0.18-0.68)$ & .002 & $0.31(0.09-1.002)$ & .05 & \\
\hline Attitude & $6.70(3.69-12.22)$ & $<.001$ & $6.12(2.91-12.88)$ & $<.001$ & \\
\hline Subjective norm & $3.38(2.27-5.03)$ & $<.001$ & $1.14(1.13-4.06)$ & .008 & \\
\hline Perceived behavioral control & $0.84(0.64-1.09)$ & .19 & $0.46(0.27-0.80)$ & .005 & \\
\hline
\end{tabular}

${ }^{a}$ Unadjusted $\mathrm{OR}=$ Odds ratios resulting from univariate analysis of each independent variable

${ }^{\mathrm{b}}$ Adjusted OR=Odds ratios resulting from multivariable analysis in which every independent variable was included in the model

manner. Construction of beliefs and associated outcome evaluations in ethically and legally gray scenarios is arguably very difficult, even for experienced pharmacists. Previous research with pharmacists and prescribers indicates default substance abuse-related reactions and behaviors are routinely not in the patient's best interest. ${ }^{17}$

Student pharmacists may model their decision-making processes on observations of preceptors and supervisors. Similar to licensed pharmacists, learners may be prone to engaging in behaviors that are not in the best interest of their patients if they reflexively construct incomplete lists of beliefs and outcome evaluations (and thus their attitudes) in their minds. In an assessment of students' attitudes and responses to practice-based ethical dilemmas, Myers and colleagues identified considerable uncertainty regarding dispensing decisions and high levels of perceived difficulty in responding to ethical dilemmas. ${ }^{22}$ Evaluating students' attitudes, subjective norm beliefs, and perceived behavioral control beliefs can guide development of efficient and effective interventions that inform and modify students' default beliefs and behaviors. In the current study, emphasizing students' self-reflection on beliefs and outcome evaluations through learning experiences, including listing of all beliefs, could encourage students to thoroughly construct and consider their attitudes. Likewise, engaging students 


\section{American Journal of Pharmaceutical Education 2019; 83 (5) Article 6767.}

Table 3. Output From Negative Binomial Regression Showing Factors That Influence Pharmacy Students' Intent to Dispense in 10 Scenarios Similar to Substance Abuse-Related Gray Areas of Pharmacy Practice (N=159)

\begin{tabular}{|c|c|c|c|}
\hline Variables & b (standard error) & $e^{b}$ & $p$ Value \\
\hline \multicolumn{4}{|l|}{ Early Refill Scenario } \\
\hline Gender (male vs female) & $0.48(0.23)$ & 1.62 & .03 \\
\hline Prior work experience (yes vs no) & $-0.21(0.28)$ & 0.81 & .47 \\
\hline Cohort (2016 vs 2017) & $-0.10(0.24)$ & 0.91 & .69 \\
\hline Attitude & $0.44(0.09)$ & 1.55 & $<.001$ \\
\hline Subjective norm & $0.12(0.10)$ & 1.13 & .22 \\
\hline Perceived behavioral control & $-0.20(0.10)$ & 0.82 & .05 \\
\hline Intercept & $-0.14(0.74)$ & - & .85 \\
\hline \multicolumn{4}{|l|}{ Buprenorphine Scenario } \\
\hline Gender (male vs female) & $-0.11(0.22)$ & 0.90 & .61 \\
\hline Prior work experience (yes vs no) & $0.16(0.29)$ & 1.18 & .57 \\
\hline Cohort (2016 vs 2017) & $-0.24(0.23)$ & 0.79 & .29 \\
\hline Attitude & $0.34(0.09)$ & 1.40 & $<.001$ \\
\hline Subjective norm & $0.11(0.09)$ & 1.11 & .23 \\
\hline Perceived behavioral control & $0.06(0.11)$ & 1.06 & .59 \\
\hline Intercept & $-0.97(0.84)$ & - & .25 \\
\hline \multicolumn{4}{|l|}{ Pain Clinic Scenario } \\
\hline Gender (male vs female) & $0.16(0.13)$ & 1.17 & .21 \\
\hline Prior work experience (yes vs no) & $0.18(0.16)$ & 1.20 & .25 \\
\hline Cohort (2016 vs 2017) & $0.05(0.13)$ & 1.05 & .73 \\
\hline Attitude & $0.45(0.06)$ & 1.57 & $<.001$ \\
\hline Subjective norm & $0.10(0.06)$ & 1.10 & .10 \\
\hline Perceived behavioral control & $-0.11(0.05)$ & 0.90 & .02 \\
\hline Intercept & $-0.67(0.40)$ & - & .09 \\
\hline \multicolumn{4}{|l|}{ Syringe Scenario } \\
\hline Gender (male vs female) & $-0.01(0.12)$ & 0.99 & .95 \\
\hline Prior work experience (yes vs no) & $0.05(0.15)$ & 1.05 & .73 \\
\hline Cohort (2016 vs 2017) & $0.05(0.13)$ & 1.05 & .71 \\
\hline Attitude & $0.42(0.05)$ & 1.53 & $<.001$ \\
\hline Subjective norm & $0.02(0.04)$ & 1.02 & .69 \\
\hline Perceived behavioral control & $-0.06(0.05)$ & 0.94 & .20 \\
\hline Intercept & $-0.08(0.40)$ & - & .84 \\
\hline \multicolumn{4}{|l|}{ Pseudoephedrine Scenario } \\
\hline Gender (male vs female) & $0.01(0.08)$ & 1.01 & .94 \\
\hline Prior work experience (yes vs no) & $0.28(0.10)$ & 1.32 & .003 \\
\hline Cohort (2016 vs 2017) & $-0.10(0.08)$ & 0.90 & .18 \\
\hline Attitude & $0.31(0.03)$ & 1.36 & $<.001$ \\
\hline Subjective norm & $0.11(0.04)$ & 1.12 & .002 \\
\hline Perceived behavioral control & $-0.06(0.03)$ & 0.94 & .04 \\
\hline Intercept & $-0.20(0.27)$ & - & .46 \\
\hline
\end{tabular}

Note: $\mathrm{e}^{\mathbf{b}}$ denotes e to the power of the logistic coefficient (b) from the binomial logistic regression

in a systematic approach to ethical decision-making, such as that proposed by Buerki and Vottero, could facilitate more thorough case consideration. ${ }^{23}$ Given the extent to which attitudes predicted dispensing behaviors in our study, implementing learning activities that emphasize attitude deconstruction and (re)formation, as opposed to spending time on subjective norms and perceived behavioral control beliefs, would be warranted to maximize impact and efficiency. Likewise, providing students the opportunity in an educational setting to struggle with gray scenarios, including the interpersonal communication inherent in most scenarios, may reconcile differences between what they have been taught and what has been modeled for them, leading to improved decision-making and communication.

There are several limitations to this study. Despite employing commonly used TPB items, poor reliability in the internal consistency of perceived behavioral control 


\section{American Journal of Pharmaceutical Education 2019; 83 (5) Article 6767.}

items limited statistical modeling for and interpretation of that construct. Likewise, other factors not captured explicitly by the TPB may influence ethical decisionmaking. Fear of patient response, for example, may inform perceived behavioral control beliefs or may not have been considered by students. Future research is warranted to explore additional factors that may influence dispensing behaviors. Limitations inherent in the self-reporting of behavioral intent in a written case scenario, especially among students with limited pharmacy experience, are also present. Efforts were taken to account for co-curricular pharmacy work experience. Even though the surveys were anonymous and completed individually, the potential for social desirability bias must be considered. Given that this study was conducted in two student cohorts at one institution, the results may not be generalizable to other student cohorts and to decision-making in actual pharmacy practice and different localities where pharmacy laws may differ. Whereas the laboratory could be considered an intervention, the effectiveness of the laboratory in changing students' behaviors and perceptions was not assessed because of time limitations.

\section{CONCLUSION}

Pharmacy students' attitudes consistently predicted their intent to dispense across five ethically and legally gray community pharmacy practice scenarios involving potential substance misuse or abuse. These findings can inform development of interventions that provide students with opportunities to practice decision-making while simultaneously developing awareness of upstream factors that drive downstream behaviors. Engaging pharmacy students in such interventions during the professional program could positively influence their decision-making in gray areas of practice prior to entering practice and possibly negatively impacting patient care. Future TPB-based studies that evaluate the influence of interventions on students' perceptions and behaviors are warranted.

\section{REFERENCES}

1. Center for Behavioral Health Statistics and Quality. Results from the 2017 National Survey on Drug Use and Health: Detailed Tables. Substance Abuse and Mental Health Services Administration. September 2018.

2. Zibbell JE, Iqbal K, Patel RC, et al. Increases in hepatitis $\mathrm{C}$ virus infection related to injection drug use among persons aged $</=30$ years - Kentucky, Tennessee, Virginia, and West Virginia, 20062012. MMWR Morb Mortal Wkly Rep. 2015;64(17):453-458.

3. U.S. Departmentof Health and Human Services (HHS) OotSG. Facing Addiction in America: The Surgeon General's Report on Alcohol, Drugs, and Health. Washington, DC. November 2016. 4. Volkow ND, Collins FS. The role of science in addressing the opioid crisis. N Engl J Med. 2017;377(4):391-394.
5. ASHP statement on the pharmacist's role in substance abuse prevention, education, and assistance. Am J Health Syst Pharm. 2016;73(9):e267-270.

6. American Association of Colleges of Pharmacy. Substance Abuse: The Pharmacy Educator's Role in Prevention and Recovery. June 21, 2013.

7. Ninety-four pharmacy schools publicly commit to educating students on prevention and treatment of prescription drug abuse [press release]. American Association of Colleges of Pharmacy. June 2, 2016.

8. Fleming ML, Barner JC, Brown CM, Shepherd MD, Strassels S, Novak S. Using the theory of planned behavior to examine pharmacists' intention to utilize a prescription drug monitoring program database. Res Social Adm Pharm. 2014;10(2):285-296. 9. Hagemeier NE, Murawski MM, Lopez NC, Alamian A, Pack RP. Theoretical exploration of Tennessee community pharmacists' perceptions regarding opioid pain reliever abuse communication. Res Social Adm Pharm. 2014;10(3):562-575.

10. Cochran G, Field C, Lawson K. Pharmacists who screen and discuss opioid misuse with patients: future directions for research and practice. J Pharm Pract. 2015;28(4):404-412.

11. Wright RE, Reed N, Carnes N, Kooreman HE. Concern about the expanding prescription drug epidemic: a survey of licensed prescribers and dispensers. Pain Physician. 2016;19(1):E197-208. 12. Neville MW. Pharmacy students' attitudes about treating patients with alcohol addiction after attending a required mutual support group. Am J Pharm Educ. 2014;78(2):Article 39.

13. Richard MD, Brahm NC, Davis TS. Professional development: Students' perceptions of an immersion elective in substance abuse. Curr Pharm Teach Learn. 2013;5:54-61.

14. Cochran G, Field C, Lawson K, Erickson C. Pharmacists' knowledge, attitudes and belief regarding screening and brief intervention for prescription opioid abuse: a survey of Utah and Texas pharmacists. J Pharm Health Serv Res. 2013;4(2):71-79. 15. Gavaza P, Fleming M, Barner JC. Examination of psychosocial predictors of Virginia pharmacists' intention to utilize a prescription drug monitoring program using the theory of planned behavior. Res Social Adm Pharm. 2014;10(2):448-458.

16. Fleming ML, Barner JC, Brown CM, Shepherd MD, Strassels SA, Novak S. Pharmacists' training, perceived roles, and actions associated with dispensing controlled substance prescriptions. $J$ Am Pharm Assoc. 2014;54(3):241-250.

17. Hagemeier NE, Tudiver F, Brewster S, Hagy EJ, Hagaman A, Pack RP. Prescription drug abuse communication: a qualitative analysis of prescriber and pharmacist perceptions and behaviors. Res Social Adm Pharm. 2016;12(6):937-948.

18. Ajzen I. The theory of planned behavior. Organizational Behavior and Human Decision Processes.1991;50:179-211.

19. Tai BW, Hata M, Wu S, Frausto S, Law AV. Prediction of pharmacist intention to provide medication disposal education using the theory of planned behaviour. J Eval Clin Pract. 2016;22(5):653-661.

20. Ajzen I. Constructing a theory of planned behavior questionnaire. 2010. http://people.umass.edu/aizen/pdf/ tpb.measurement.pdf. Accessed November 12, 2012.

21. Tennessee Board of Pharmacy. 1140-03.10: Conditions for Delivery or Sale. 2017.

22. Myers JR, Kiersma ME, Plake KS. Assessment of student pharmacists' ethical decision-making. Curr Pharm Teach Learn. 2017;9(6):996-1002.

23. Baker DM, Buerki RA, Vottero LD. Pharmacy Ethics: A Foundation for Professional Practice. Washington, DC: American Pharmacists Association; 2013. 